\title{
OBJECIÓN DE CONCIENCIA Y SALUD REPRODUCTIVA EN CHILE: DOS CASOS PARADIGMÁTICOS
}

\author{
Lidia Casas Becerra* y Claudia Dides Castillo**
}

Resumen: Este artículo reflexiona sobre objeción de conciencia y salud reproductiva en dos casos paradigmáticos: la anticoncepción de emergencia y la esterilización voluntaria. Se plantean varias preguntas respecto de la objeción de conciencia en el campo de la salud reproductiva y de los dilemas éticos (y políticos) de los cuales, como sociedad democrática, debemos hacernos cargo.

Palabras clave: objeción de conciencia, salud reproductiva, esterilización voluntaria, anticoncepción de emergencia y derechos

\section{CONSCIENTIOUS OBJECTION AND REPRODUCTIVE HEALTH IN CHILE: TWO PARADIGMATIC CASES}

Abstract: This paper meditates upon conscientious objection and reproductive health in two paradigmatic cases: emergency contraception and voluntary sterilization. Several questions arise regarding conscientious objection towards reproductive health and its ethical (and political) dilemma. As a democratic society we should be concerned about both of them..

Key words: conscientious objection, reproductive health, voluntary sterilization, emergency contraception and human rights

\section{OBJEÇÃO DE CONSCIÊNCIA E SAÚDE REPRODUTIVA NO CHILE: DOIS CASOS PARADIGMÁTICOS}

Resumo: Este artigo reflete sobre a objeção de consciência e saúde reprodutiva nos dois casos paradigmáticos: a anticoncepção de emergência e a esterilizaçáo voluntária. Formulam-se uma serie de perguntas a respeito da objeçáo de consciência no campo da saúde reprodutiva e dos dilemas éticos e políticos dos quais, como sociedade democrática, devemos nos responsabilizar.

Palavras chave: objeção de consciência, saúde reprodutiva, esterilização voluntária, anticoncepção de emergência e direitos

* Abogada. Facultad de Derecho, Universidad Diego Portales, Chile Correspondencia: lidia.casas@udp.cl

Socióloga. Programa Equidad de Género y Políticas Públicas, Facultad Latinoamericana de Ciencias Sociales, FLACSO, Chile

Correspondencia: cdides@flacso.cl 


\section{Introducción}

Desde los años 90 hemos asistido en Chile a una serie de debates públicos sobre temas asociados a salud sexual y reproductiva, y a derechos sexuales y reproductivos: la educación sexual (en particular, las Jornadas de Conversación sobre Afectividad y Sexualidad, JOCAS), las campañas de prevención del SIDA, la esterilización voluntaria, la prevención del embarazo adolescente como acciones de la política pública, las conferencias internacionales (Conferencia Internacional de Población y Desarrollo de El Cairo, 1994, Cuarta Conferencia Mundial de Naciones Unidas sobre la Mujer de Beijing, 1995) -frente a las cuales el Estado ha tenido que pronunciarse internacionalmente-, el proyecto ley marco sobre derechos sexuales y reproductivos como una propuesta por parte de la sociedad civil, el aborto como un problema de salud pública, la homosexualidad como un tema social, entre otros(1).

Dos iniciativas de política pública han causado mayor polémica en los últimos cinco años y han repercutido en el acceso inequitativo de las mujeres al uso de tecnologías anticonceptivas: por un lado, la introducción, autorización y comercialización de la "píldora del día después", o anticoncepción de emergencia, y, por otro, la normativa de esterilización voluntaria $(2)^{1}$.

Estos debates han tenido lugar en un contexto socioeconómico político y cultural de grandes transformaciones, con avances en materia económica y mayor bienestar en amplios sectores de una población que ha modificado sus creencias, sistemas de valores, estilos de vida y construcciones subjetivas, en un proceso - no lineal ni homogéneo- que es parte de la modernidad. Este escenario exige a los individuos construir una identidad propia en un mundo que ofrece múltiples opciones, generando mayor libertad y, a la vez, más incertidumbre(3).

\section{Antecedentes históricos para comprender el debate en Chile}

La modernidad y sus procesos de democratización han puesto en primer plano la temática de la ciudadanía

1 En distintos países de la región se han promovido acciones cautelares, normalmente de orden constitucional, para detener el registro o la comercialización de la anticoncepción de emergencia, tales son los casos seguidos en Argentina, Colombia, México, Ecuador y Chile. Sólo en el caso peruano se ha visto una discusión política que ha incluido a buena parte de las instituciones de ese país, y que culminó con el pronunciamiento de un fallo favorable en el Tribunal Constitucional en 2006. y de su ejercicio en los procesos de desarrollo de las sociedades modernas, proceso que también se ha extendido al ámbito de la sexualidad y la reproducción. Se ha producido la separación entre sexualidad y reproducción a partir del acceso de las mujeres a las tecnologías anticonceptivas y la posibilidad de elegir en qué momento tener hijos. La principal consecuencia ha sido la liberalización de las prácticas sexuales. Sin embargo, en este marco de apertura, también se han consolidado grandes corrientes conservadoras. La disputa político-ideológica en torno a las tecnologías anticonceptivas no es nueva. Diversos antecedentes históricos-en nuestro país y en el resto del mundo-dan cuenta de las tensiones que conlleva este tema.

Zárate señala que, a fines del siglo XIX, las nocivas condiciones laborales y las enfermedades afectaron la salud de niños y mujeres de tal manera que médicos higienistas y feministas de la época impulsaron medidas de prevención social que redujeran los costos asociados a la urbanización e industrialización que experimentaba el país(4). Se elaboró entonces un discurso, que se materializó en el siglo XX, cuyo objetivo era colaborar en la tarea primordial de las madres: la dedicación completa a sus hijos y su posterior materialización en políticas. "La vocación por difundir la llamada 'maternidad científica' y la protección a las mujeres comenzó con el interés de la comunidad erudita, específicamente los médicos que atendían a estas mujeres en dispensarías, casas de maternidad o de huérfanos y más tarde en los servicios hospitalarios" (4, p. 170). La maternidad en Chile dejaba de ser una práctica íntima y privada para transformarse en una responsabilidad de carácter social(1).

En Chile, los movimientos con un discurso elaborado y con audiencias masivas por la regulación de la fecundidad se inician con la creación del Movimiento Pro Emancipación de la Mujer (MEMCH). Hacia los años 30, las mujeres de estratos populares recurrían al aborto y a prácticas mágicas como métodos anticonceptivos. El MEMCH fue probablemente influido por los movimientos de opinión organizados para promover la planificación familiar en Estados Unidos y Europa. A su vez, influyó la crisis económica mundial de los 30, dejando efectos desastrosos en la calidad de vida de la población. Ya en esos años se proponía el mejoramiento de los "salarios femeninos, proteger a la madre soltera, admitir en los casos pertinentes el divorcio con disolución de vínculo, promover una educación sexual científica y sin falsa moral, y terminar 
con el complejo de inferioridad que la sociedad había inculcado a las mujeres, todo lo cual haría posible acabar con la prostitución, otro de los grandes males de la época" $(1, p .104)$.

Illanes sostiene que ya a comienzos de los años 60 la Iglesia Católica venía preocupándose del control de la natalidad. Su postura oficial prohibía el uso de cualquier "tipo de anticonceptivo; no obstante, teólogos y autoridades más liberales y progresistas fueron sacando a la luz nuevas fuentes doctrinarias que permitían abrirse a su 'aceptación': era una postura relativa, disidente" (5). Hasta 1963 duraron las acciones de regulación de la natalidad que habían sido incorporadas a los programas del Servicio Nacional de Salud (SNS). Sin embargo, se continuó trabajando en ello con finalidades "asistenciales, de investigación y adiestramiento", en coordinación con universidades nacionales y con agencias internacionales (Ford Foundation, Population Council, Public Welfare Foundation, Harvard University)(5).

El control de la natalidad coincidía con una política de desarrollo económico y social para la estabilidad política del continente, propiciada por Estados Unidos. "Cinco dólares gastados en control de la natalidad producen más para el desarrollo que 95 dólares en inversiones y desarrollo de servicios" $(5$, p. 460).

El SNS siguió realizando acciones de control de natalidad, al margen de la propia estructura administrativa del servicio. En 1967, la Iglesia Católica reiteraba su oposición terminante al uso de anticonceptivos farmacéuticos y mecánicos. En respuesta, el director de entonces explicaba que el SNS proponía métodos de control a casos médicamente específicos, "pero en ningún caso plantea un control de la natalidad en Chile". Esto correspondía a decisiones de política de "más arriba". Reiteraba un principio intransable del servicio respecto de las acciones realizadas: "nosotros tenemos otras razones para el control limitado de la natalidad. Cuatrocientas mujeres mueren y 50.000 son hospitalizadas por consecuencias del aborto criminal" ( 5 , p. 461). Como plantea Illanes, ni la Ley ni la Iglesia cedieron: tampoco el SNS. Este siguió desarrollando su acción como respuesta a un encuentro tácito y definitivo entre la ciencia y la sociedad $(5, p .461)$.

En 1969, profesionales de la salud del área sur de Santiago dieron a conocer a la comunidad nacional los promisorios resultados de la labor del SNS en control de natalidad en la población San Gregorio de la comuna de
La Granja, con una de las más altas tasas de fecundidad y de aborto. Sobre la base de una muestra, que incluyó 1.293 mujeres entre 15 y 44 años (un 20\% de la población), se demostró una clara disminución de la tasa de fecundidad y de abortos después de un año de aplicado el programa en los ámbitos educativo y asistencial. Se llegó a establecer que el aborto disminuyó en un $40 \%$ y la fecundidad en un $20 \%$ en ese período de un año(1).

En estos mismos años, por influencia de la Federación Internacional de Planificación Familiar (IPPF), se creó el Comité de Protección de la Familia, cuyo objetivo fue reducir las elevadas tasas de natalidad, la mortalidad materna y la letalidad por aborto(5). A fines de 1965, el gobierno de Frei Montalva le otorgó personalidad jurídica, formándose la Asociación Chilena de Protección a la Familia (APROFA). Posteriormente, este mismo gobierno definió una política pública de paternidad responsable(6), cuya implementación estuvo marcada por la pugna de los poderes eclesiásticos, médicos y políticos(7).

Una de las reacciones más importantes de la época, que da cuenta de la disputa política ideológica de aquellos años, provino de la Santa Sede, sintetizada en la Encíclica sobre la Vida Humana (1968).

Al implementarse su acceso en forma masiva, la planificación familiar estaba precedida de objetivos "técnicos" más que de un lenguaje de derechos, es decir, de la capacidad de las personas de decidir por sí mismas ser madres o padres y cuántos hijos deseaban tener. El debate estuvo teńido por consideraciones biomédicas sobre el contexto sanitario de la época: altos índices de abortos y muertes por esa causa(8). La planificación familiar, sin embargo, garantizó el uso de métodos anticonceptivos a la mayoría de la población del país, lo que constituyó un paso fundamental para la autonomía reproductiva de las mujeres.

\section{Objeción de conciencia y salud reproductiva}

La literatura alude a la objeción de conciencia como una manifestación de desobediencia civil $(9)^{2}$, pero es distinta de ésta. Peces-Barba sostiene que la objeción es

2 La Evangelium Vitae enseña la objeción de conciencia a leyes civiles moralmente inaceptables, cuando en verdad se trataría de una desobediencia civil, pues intenta revertir y modificar aquellas leyes que repugnan al evangelio católico. John Rawls señala que muchas veces es difícil dilucidar en términos prácticos cuándo se puede hablar de desobediencia civil o de objeción de conciencia. 
una inmunidad o un derecho subjetivo, y supone una excepción a una obligación jurídica(10). La objeción supondría la regulación de la exención de cumplimiento de una obligación jurídica fundamental, derivada normalmente de las relaciones laborales o funcionarias (contrato de trabajo o estatuto funcionario); está dirigida a los poderes públicos y a los particulares y siempre se plantea frente a una obligación personal. El accionar del objetor responde únicamente a dejar a salvo su conciencia, ya sea por razones religiosas o filosóficas.

La entrega de servicios de salud en el ámbito de la reproducción y sexualidad puede pugnar con las convicciones de quien debe otorgarlos. Quien debe ejecutar una acción se ve enfrentado con su conciencia y con los deberes profesionales, legales o funcionarios. En muchos países se recoge la objeción de conciencia en una legislación específica o bien se desprende de las garantías constitucionales.

En los países occidentales, la objeción de conciencia ha surgido tradicionalmente frente a la realización de abortos no punibles, la esterilización y, recientemente, frente al uso de técnicas de fertilización asistida o maternidad sustituta(11). La relación entre salud sexual y reproductiva y la objeción aparece más o menos nítida en la medida en que la primera entraña un conjunto de condiciones y presupuestos que podrían contener visiones éticas contrapuestas.

La salud sexual y reproductiva es un completo estado de bienestar físico, mental y social -y no la mera ausencia de enfermedades o dolencias-. Supone el derecho de las personas para reproducirse y regular su fecundidad, y que las mujeres no se vean expuestas a riesgos innecesarios frente al embarazo y parto, pudiendo disfrutar de su vida sexual sin riesgos(11, p. 11). El concepto mismo alude a una serie de condiciones para alcanzar ese estado e implica el ejercicio de derechos.

Las decisiones sobre reproducción comprometen la autonomía de las personas, en particular de las mujeres, con el fin de que puedan definir su propio plan de vida. La decisión de una mujer $\mathrm{u}$ hombre que desea poner fin a su vida reproductiva a través de la esterilización se contrapone con las convicciones personales de un profesional que sólo intervendría esa capacidad por razones médicas y no por motivos de autonomía.

\section{La incorporación de la anticoncepción de emergencia en Chile: el contexto}

La introducción de este fármaco se enmarca en un contexto sociocultural político y económico que da cuenta de profundas transformaciones. Existe una tendencia a una mayor liberalización de las prácticas sexuales, especialmente entre adolescentes(12), y en el marco de cambios de los patrones tradicionales de lo masculino y lo femenino(13). Los significados y las prácticas sexuales de mujeres y hombres ya no responden al "modelo" que formó parte por décadas del sentido común(13).

La aceptación, masificación y legitimación de los métodos de regulación de natalidad se expresan en los perfiles decrecientes del ritmo de crecimiento demográfico en el país, pero también en las opiniones reveladas por diferentes encuestas. Según la del Grupo Iniciativa (1999), la mayoría está de acuerdo con el derecho de todas las personas a usar anticonceptivos dentro o fuera del matrimonio (9 de cada 10). Esta proporción se incrementa entre las jóvenes de 20 a 33 años(14). Estudios realizados en Chile muestran mayor valoración de la necesidad de la educación sexual, protección de las relaciones sexuales, prevención y control del embarazo adolescente, prevención del VIH/SIDA, el rechazo de toda violencia de género, entre otras prioridades.

En 1996, el Instituto Chileno de Medicina Reproductiva (ICMER) identificó la necesidad de incorporar la anticoncepción de emergencia(AE) $(15)^{3}$ como política pública. Para ello, realizó una investigación cualitativa(16), cuyo objetivo fue evaluar la aceptabilidad y los aspectos legales de la $\mathrm{AE}$, y la situación de los derechos sexuales y reproductivos en Chile. Posteriormente, realizó un trabajo de sensibilización con las autoridades de salud sobre la importancia de la AE para reducir embarazos no deseados, particularmente en casos de agresión sexual, todo ello entendiendo que Chile no permite el aborto bajo ninguna circunstancia. El Ministerio de Salud recogió tales consideraciones en el Protocolo de Intervención en los Servicios de Urgencia para Casos de Agresiones Sexuales, de 1998, elaborado por un

3 La anticoncepción de emergencia surge en los años 60 como respuesta médica frente a las consecuencias de una agresión sexual, en particular un embarazo no deseado. En la mayoría de los países se la incluye también en las normas para la atención de los casos de violación. También ha sido conocida como "anticoncepción postcoital" y a la pastilla usada para AE se la ha llamado "pildora del día después". 
conjunto de especialistas de los servicios públicos de salud. No obstante, el ministro de la época decidió emitir una fe de erratas a la recomendación del uso de $\mathrm{AE}$, seńalando simplemente desconocerla ${ }^{4}$.

En los últimos cuatro ańos, iniciativas y procesos de política pública relativos a la $\mathrm{AE}$ han trasladado parte de la discusión al espacio judicial. La polémica en los medios de comunicación, en particular en la prensa escrita, ha estado directamente vinculada con las medidas que ha tomado el Ministerio de Salud y organismos dependientes, y con las resoluciones de los tribunales de justicia.

En el transcurso de seis años de debates públicos (20012007) se ha logrado la venta del fármaco, primero Postinor 2 y luego TACE, bajo receta médica retenida. Sin duda, su costo constituye una barrera más al acceso oportuno a un medio que, por definición, actúa más eficazmente en las primeras horas luego de una relación sexual no protegida.

La introducción de la $\mathrm{AE}$ en el sistema público ha estado plagada de problemas, tanto judiciales como de políticas públicas. Su entrega generó una reacción de un grupo de alcaldes autodenominados "objetores", quienes, desde 2004, no han cambiado su política sobre la provisión de este anticonceptivo ni el gobierno central ha exigido su cumplimiento. En este caso, no hay en verdad una objeción, sino la resistencia directa a obedecer un mandato técnico y de provisión de servicios con el propósito de revertir la política pública. Si consideramos la objeción como una inmunidad, ella sólo estaría contemplada respecto de quien tiene la obligación de prescribir, en este caso los profesionales de salud directamente involucrados, y no respecto de quien pugna por echar abajo la normativa y la política pública sobre la materia.

De ser consistentes con el lenguaje de la objeción, los alcaldes no deberían interferir en el proceso de entrega y asegurar que sus profesionales actuaran conforme a su propio marco ético. El ejercicio de la objeción implica, a su vez, la instalación de una política respetuosa, tanto de las convicciones de la población usuaria como de sus profesionales.

4 Escrito presentado por Lidia Casas y Pablo Isla a la Corte de Apelaciones, 2001, representando al Instituto Chileno de Medicina Reproductiva ICMER, la Asociación Chilena de Protección a la Familia APROFA y la Corporación de Salud y Políticas Sociales CORSAPS.

\section{La esterilización voluntaria}

La modificación de la regulación sobre esterilización voluntaria se gesta en un contexto de demandas del movimiento de mujeres que exigen respeto y garantía a sus derechos en sus decisiones reproductivas(8). Al dictar la Resolución Exenta No 2.326, que fija directrices para los servicios de salud sobre esterilización femenina y masculina ${ }^{5}$, la autoridad dio cumplimiento a la Convención sobre Eliminación de todas las Formas de Discriminación contra la Mujer, ratificada en 1989, y a los acuerdos suscritos por Chile en la Conferencia sobre la Mujer (Beijing 1995). Esta Resolución regula la esterilización voluntaria de las mujeres y permite la realización de vasectomías (procedimiento que antes no estaba reglamentado ni asegurado) en establecimientos dependientes de los servicios públicos de salud, derogando todas las disposiciones discriminatorias por género y edad.

La normativa anterior, de 1975, establecía que las mujeres podían esterilizarse siempre que tuvieran una condición médica que lo justificara, tuvieran más de 32 años y 4 hijos vivos. El requisito de edad se reducía a 30 años si existía riesgo obstétrico. Además, los jefes de servicios gineco-obstétricos exigían el consentimiento del marido; cuando la mujer era separada o convivía, se solicitaba la autorización de la pareja, independientemente del estado civil. Esta norma sólo se aplicaba en los centros de salud públicos, con lo cual se producía una discriminación por razón de la clase social(8).

En síntesis, la nueva normativa estipula que se puede acceder a la esterilización a petición de la persona solicitante, por recomendación médica o a solicitud de terceros, en casos especiales. Es una decisión personal y emana de la voluntad libre manifestada por quien lo solicite, sin que ello quede supeditado a la aprobación de terceras personas, en el caso de mayores de edad en posesión de sus facultades mentales. Antes de la esterilización, el profesional tratante -médico o matronadebe entregar consejería en salud sexual y reproductiva a la persona, con información completa de métodos anticonceptivos y sobre la situación de irreversibilidad, en caso de vasectomía, y de alta improbabilidad de reversión, en caso de esterilización femenina, incluyendo las posibles complicaciones y porcentaje de fracaso de

5 Fue dictada por el Ministerio de Salud de Chile el 30 de noviembre de 2000 . 
ambas. Si el solicitante persiste en su decisión, se dejará constancia previa en un documento. La persona podrá desistirse antes de iniciado el proceso, bastando para ello su mera declaración en tal sentido. En caso de que se vaya a practicar la esterilización durante la realización de una operación cesárea, se deben efectuar antes los procedimientos de consejería. Para la esterilización de personas en edad reproductiva que padecen de una enfermedad discapacitante que produzca carencia de discernimiento, se requiere el consentimiento del apoderado del paciente que ratifique la opinión del médico tratante.

Hasta 2000, el promedio anual de esterilizaciones quirúrgicas femeninas era de $15 \mathrm{mil}$ al año, y en 2006 subió a alrededor de 21 mil. El mayor aumento se produjo en la salud pública, donde hubo $18 \mathrm{mil}$ intervenciones, mientras que en los centros hospitalarios privados sólo hay registrados 2.811 casos(17). Según un informe del Ministerio de Salud, en 2000 se realizaron 16.344 esterilizaciones femeninas y 137 vasectomías, lo que representa el 99,2\% y el 0,8\% del total de esterilizaciones, respectivamente(18). Este fue el primer ańo en el cual se realizaron vasectomías en el sistema público de salud.

Este diagnóstico reportó que el total de servicios de salud (28) realizaron esterilizaciones femeninas y sólo 9 efectuaron vasectomías. Además, no todos ellos contaban con información sobre el número de solicitudes de esterilización femenina, lo que no permitió conocer cuál era el nivel de demanda insatisfecha para este procedimiento en estos servicios. De los servicios que contaban con esta información (21), sólo tres dieron respuesta a la demanda de esterilización en un ciento por ciento de las solicitudes (Servicios de Salud Aysén, Araucanía Norte y Bío-Bío). Los servicios con más alta demanda insatisfecha fueron los de Arica (45\%), Metropolitano Norte (56\%) y Metropolitano Oriente (57\%). En relación con la esterilización masculina, sólo seis servicios registraron el número de solicitudes de vasectomía, por lo que no es posible conocer el nivel de demanda de este procedimiento ni su grado de respuesta por parte de los servicios. El mayor número de vasectomías se realizó en los servicios de salud Valparaíso (48\%) y Metropolitano Norte (28\%)(18).

Las causas más frecuentes para no realizar las esterilizaciones solicitadas fueron la falta de horas médicas de anestesistas y gineco-obstetras, la falta de horas de pabellón, la ausencia de implementación de pabellones de cirugía laparoscópica y el rechazo por exigirle a las mujeres los requisitos de la normativa anterior (edad y número de hijos, por ejemplo)(18, p. 47).

\section{Tensiones entre objeción de conciencia y salud reproductiva}

Tanto la esterilización voluntaria como la anticoncepción de emergencia -en cuanto tecnologías anticonceptivas- son un derecho para mujeres y hombres. En las últimas décadas hemos asistido a diferentes cambios sociodemográficos y normativos, influenciados por un fenómeno de gran relevancia: se ha modificado el valor social asignado a la sexualidad y a la reproducción. A partir de los diferentes procesos de modernización, ha surgido un elemento central para posibilitar el ejercicio de los derechos en este campo, se trata de la separación entre sexualidad y reproducción o, como lo llama Montaño(19), la separación entre erotismo y fertilidad.

Este proceso va ligado al acceso a los métodos de regulación de la fecundidad y a la implementación de programas de salud reproductiva en el ámbito público, repercutiendo en el proceso de toma de decisiones de las mujeres respecto a su sexualidad y reproducción. Según Dides(1), se trata de un nuevo camino de búsqueda de autonomía de las mujeres y, por tanto, un cuestionamiento a los sistemas de dominación de género en estos campos. Pero, también, ha repercutido en diversos debates sociales que muchas veces esconden concepciones del orden social, su institucionalización y sus regulaciones. En la medida en que el debate se enfoque sólo en aspectos técnicos o biomédicos, se elude una discusión vigorosa sobre el contenido y alcance de los derechos involucrados(20).

La separación entre sexualidad y reproducción ha dado paso a la construcción de los denominados "derechos sexuales y reproductivos", que buscan refundar la relación entre lo público y lo privado en forma de un cambio de paradigma. Se definen como derechos y libertades fundamentales de todas las personas -sin discriminación-, que permiten adoptar libremente, sin ningún tipo de coacción o violencia, una amplia gama de decisiones sobre aspectos consustanciales a la vida humana. Su ejercicio implica contar con información y acceso a los servicios y medios que se requieren para ejercer estas decisiones. También conlleva el derecho a tener la posibilidad de alcanzar el máximo de placer, bienestar y felicidad posibles(21). En este sentido, la 
anticoncepción de emergencia y la esterilización voluntaria son un gran avance para el ejercicio de estos derechos, porque permiten tomar decisiones autónomamente para evitar un embarazo no deseado.

La vida personal de las mujeres ha entrado en un campo de negociación de la intimidad; se ha convertido en un proceso personal y abierto que ha creado nuevas demandas. En general, los procesos de liberalización e introducción de nuevos principios, valores y discursos que ordenan la relación entre cuerpo, sexualidad y reproducción se ven enfrentados a nuevos nudos problemáticos.

Según Dides(1), los avances tecnológicos en materia de reproducción implican nuevos campos de conflicto, especialmente valóricos, en la medida en que ponen en cuestión las formas tradicionales de entender instituciones sociales básicas y principios de filiación. Chile vive un proceso de doble agenda: por una parte, un elevado estándar de vida en términos de consumo material e infraestructura, así como en demandas e intereses sociales; por otra, déficit y necesidades propias de un país subdesarrollado del tercer mundo.

La utilización de un lenguaje normativo con alto poder de persuasión moral detrás de los derechos humanos, invocando la objeción de conciencia para no realizar ciertos deberes profesionales, esconde una parte significativa de esta tensión. Los derechos entre profesionales y usuarios se enfrentan, dejando a estos últimos ausentes de la resolución del conflicto y como si ello fuera sólo un accidente. Los usuarios del sistema de salud deben ser protegidos en sus derechos. Se deben buscar fórmulas transparentes, intentando maximizar ambos actores y evitando la suma cero. Pero, ante el límite y sin otra opción, los prestadores de salud deberían hacer honor a sus responsabilidades funcionarias y profesionales.

\section{Referencias}

1. Dides C. Voces de emergencia: el discurso conservador y la píldora del día después. Santiago de Chile: FLACSO-Chile, UNFPA; 2006.

2. Casas L. La batalla de la píldora. El acceso a la anticoncepción de emergencia en América Latina. Derecho y Humanidades 2004; 10: 187-203.

3. Beck U, Giddens A, Lash S. Modernización reflexiva. Politica, tradición y estética en el orden social moderno. Madrid: Alianza Editorial; 1997.

4. Zárate MS. Proteger a las madres: origen de un debate público, 1870-1920. En: Monográficas 1, Nomadias. Santiago de Chile: Programa de Género y Cultura en América Latina (PGAL), Facultad de Filosofía y Humanidades, Universidad de Chile; 1999: 169.

5. Illanes MA. En el nombre del Pueblo, del Estado y de la Ciencia. Historia Social de la Salud Pública, Chile 1880-1973. Santiago de Chile: Colectivo de Atención Primaria; 1993: 459.

6. Jiles X, Rojas C. De la miel a los implantes. Historia de las políticas de regulación de la fecundidad en Chile. Santiago de Chile: Corporación de Salud y Políticas Sociales CORSAPS; 1992.

7. Dides C, et al. La política de fecundidad en el Chile actual: docilización o autonomización. En: Anuario del Programa de Género y Cultura. Santiago de Chile: Facultad de Filosofía y Humanidades, Universidad de Chile; 1996.

8. Casas L. ¿Del control a la autonomía? Informe de Investigación. Santiago de Chile: Facultad de Derecho, Universidad Diego Portales; 2004: 5-6.

9. Rawls J. Teoría de la Justicia. México DF: Fondo de Cultura Económica; 2002: 335-338.

10. Peces-Barba G. Derecho y derechos fundamentales. Madrid: Centro de Estudios Constitucionales; 1993: 384-388.

11. Cook R, Dickens B, Fathalla M. Salud reproductiva y derechos humanos. Integración de la medicina, la ética y el derecho. Bogotá: Oxford y Profamilia; 2003: 133.

12. Ministerio de Salud. Estudio nacional de comportamiento sexual. Santiago de Chile: MINSAL; 2000.

13. Sharim D, et al. Los discursos contradictorios de la sexualidad. Santiago de Chile: Ediciones Sur, Colección Estudios Sociales; 1996: 83.

14. Grupo Iniciativa. Encuesta nacional: Opinión y actitudes de las mujeres chilenas sobre las condiciones de género. Santiago de Chile; 1999.

15. Díaz S, Croxatto HB. Anticoncepción hormonal de emergencia. Instituto Chileno de Medicina Reproductiva. 
Actualización de: Díaz S, Croxatto HB. Anticoncepción de Emergencia. En: Pérez Sánchez A, (ed.) Ginecología, 3a edición. Santiago de Chile: Editorial Mediterráneo; 2003: 1067-1073. (Sitio en Internet) Disponible en www. anticoncepciondeemergencia.cl

16. Díaz S, Hardy E, Alvarado G, et al. Acceptability of emergency contraception in Brazil, Chile, and Mexico. Facilitating factors versus obstacles. Cad Saúde Pública 2003; 19(6):1729-1737.

17. Rajevic P. ¿¿Por qué están colgando la opción maternal? Esterilización voluntaria: ¿Por qué aumenta en las jóvenes? Revista Mujer. La Tercera, 20 de mayo de 2007.

18. Schiappacasse V, Vidal P, Casas L, et al. Chile: Situación de salud y derechos sexuales y reproductivos. Santiago de Chile: ICMER, CORSAPS, SERNAM; 2003: 47.

19. Montańo S. Los derechos reproductivos de la mujer. En: Estudios Básicos de Derechos Humanos. San José de Costa Rica: Instituto Interamericano de Derechos Humanos; 1996.

20. Casas L, Contesse J. La pildora del día después, Derechos sexuales y reproductivos, protección de la vida y políticas públicas. Caso preparado para Casoteca Latino-Americana de Derecho y Política Pública de la Escola de Direito de Sao Paulo de la Fundación Getulio Vargas. (Sitio en Internet) Disponible en http://www.gvdireito.com.br/casoteca/casosEspanhol. aspx?PagId=DKGCTRVO

21. Dides C. Proyecto de Ley marco sobre derechos Sexuales y Reproductivos. En: Vidal F, Donoso C, (edit.) Cuerpo y Sexualidad. Santiago de Chile: Vivo Positivo, FLACSO, Universidad Arcis; 2002.

Recibido: 6 de julio de 2007.

Aceptado: 3 de agosto de 2007. 